\title{
MRS fall meeting: a hybrid conference experience
}

\author{
The Materials Research Society (MRS) fall meeting is a fixture in the conference calendar of the \\ global materials science community. This year, for the very first time, the conference went hybrid, \\ posing new opportunities, but also challenges, for organizers, speakers and attendees.
}

The Materials Research Society (MRS) fall meeting has always been a highlight for the Nature Reviews Materials team. Travelling to wintery Boston, meeting old and new colleagues and friends, and learning about the latest research in materials science - the MRS fall meeting has never disappointed. Many articles have been commissioned at this conference, and new ideas and collaborations have been formed. As for many other big and small conferences, the pandemic forced the MRS meeting to go virtual last year, offering a somewhat different conference experience. Although we may have gotten used to online conferences by now, and their benefits in terms of inclusivity are undeniable, a certain degree of online conference fatigue has settled in. Therefore, many were excited to hear that the 2021 MRS fall meeting would happen as a hybrid event, with an in-person conference in Boston, followed by a virtual event. Our team has attended both.

It was refreshing to engage $100 \%$ in an in-person conference again. As editors, our jobs centre around the passions of academics. We read a lot of papers, cover letters and peer review exchanges. And while the excitement of good science often does come through in writing, there are some experiences we have missed while stuck behind our computers. Nothing replicates the thrill (and nervousness) of witnessing a heated debate after a thought-provoking talk, or the fun of learning an author's personality after trading emails with them for years. Networking is a key aspect of scientific conferences, and we have not quite managed yet to replicate in-person networking in the virtual space. Therefore, we were thrilled to talk science again face-to-face, and enjoy spontaneous scientific exchange with the community.

However, we would be remiss to discuss this MRS meeting without discussing COVID-19, and the fact that the Omicron variant was classified as a Variant of Concern by the WHO on the Friday before the conference. Attendees had to decide at the last minute whether the trip was worth the risk - not only of catching the virus but, especially for those outside of the USA, of getting stuck because of potential travel restrictions. The schedule was in constant flux, and symposia sessions were cancelled or condensed with hours' notice. Moreover, a packed room full of people still seems scary, and fears of catching COVID-19 likely overshadowed some of the networking events. Despite all of this uncertainty, the overall experience was impressively seamless, and the MRS organizers certainly deserve recognition for pulling it off.

One of our favourite events was the poster session for future faculty candidates, a mix of a recruiting fair, networking reception and classic poster session. The excellent Women in Materials Science panel discussion was also a highlight. Although sparsely attended in person, the event was recorded and streamable on demand, opening up its impact to a much wider audience.

During the virtual week, we were impressed by the quality of the recorded in-person talks, which were thoughtfully set up with the remote audience in mind. It would have been wonderful to have access to even more recorded talks from the in-person programme, ideally subtitled for maximum accessibility. The live virtual sessions, which spanned a rich and diverse programme, were also well organized, with Zoom meetings enabling participants to see who was in the room and engage in interesting discussions. However, many live sessions attracted only a handful of attendees, perhaps because one of the main benefits of a virtual event is the ability to catch up on content outside the conference schedule. Overall, the virtual event lacked the buzz of the in-person experience.

After almost 2 years of virtual conferences, it is clear that the experience offered by large international conferences is particularly hard to replicate online. Smaller conferences are working better in the virtual world, and we expect, going forward, to see different types of events in the real and virtual worlds, each valuable in different ways. The MRS fall meeting is much better in person, but smaller workshops, including the ones MRS organizes regularly, are informative and easy to fit into busy schedules. Whatever the future of conferences, we need to be creative and keep looking for the best ways to network and communicate science. 